\title{
代谢型谷氨酸受体激动引起星形 神经胶质细胞肿胀 *
}

\author{
袁 芳 王天佑 \\ (北京市神经外科研究所, 北京 100050)
}

\begin{abstract}
摘要用 $\left[{ }^{3} \mathrm{H}\right]-3$-氧-甲基-D-葡萄糖摄取的方法测定细胞水含量, 观察谷氨酸受体 激动剂、拮抗剂对培养的星形神经胶质细胞水含量的影响, 并观察细胞内、外钻的作 用. 结果发现: $0.5,1,10 \mathrm{mmol} / \mathrm{L}$ 的谷氨酸和 $1 \mathrm{mmol} / \mathrm{L}$ 的 trans-ACPD(代谢型谷氨 酸受体激动剂) $1 \mathrm{~h}$ 均可以引起细胞的水含量增加, $1 \mathrm{mmol} / \mathrm{L}$ 的 AMPA(离子型谷氨 酸受体激动剂) 不影响细胞的水含量, $1 \mathrm{mmol} / \mathrm{L}$ 的 L-AP3 (代谢型谷氨酸受体拮抗 剂) 可以拮抗 $1 \mathrm{mmol} / \mathrm{L}$ 谷氨酸和 trans-ACPD 的作用; 撤除细胞外液的钻, 谷氨酸不 再引起细胞的水含量增加, $20 \mu \mathrm{mol} / \mathrm{L}$ 的 $\mathrm{CdCl}_{2}$ 不能减轻谷氨酸的作用, 而 300 $\mu \mathrm{mol} / \mathrm{L}$ 的 $\mathrm{CdCl}_{2}$ 及 $30 \mu \mathrm{mol} / \mathrm{L}$ 的胆罗啉 (Dantrolene) 均可以减轻谷氨酸的作用, 提示 代谢型谷氨酸受体激动引起星形细胞肿胀, 细胞内、外 $\mathrm{Ca}^{2+}$ 在谷氨酸引起的星形细 胞肿胀中起一定的作用。
\end{abstract}

\section{关镌词 谷氮酸 trans-ACPD 星形细胞 肿胀 胆罗啉 $\mathrm{Ca}^{2+}$}

谷氨酸是中枢神经系统中普遍存在的兴奋性神经递质, 在脑缺血、脑外伤的情况下其含量 急剧增高, 高浓度的谷氨酸引起星形神经胶质细胞肿胀 (简称星形细胞) ${ }^{[1]}$, 但是机制并不清 楚. 最新的电生理学、药理学以及分子生物学的研究表明, 胶质细胞上有多种类型的谷氨酸受 体 $^{[2]}$, 目前药理学家根据受体的特异性激动剂将谷氨酸受体分为离子型谷氨酸受体 (iGluR) 和 代谢型谷氨酸受体 (mGluR), 前者直接偶联离子通道, 包括 $\mathrm{N}$-甲基-D-门冬氨酸 (NMDA) 受 体、红藻氨酸 (KA)受体、 $\alpha$-氨基-3-差基-5-甲基恶唑-4-丙酸(AMPA)受体; 后者与细胞膜上的 $G$ 蛋白相偶联, 引起磷酸肌醇及其他胞内信使物质的变化, 激动剂有反式-氨基-环戊基-1,2-二羧 酸( trans-ACPD) ${ }^{[3]}$. 文献报道使君子酸可以引起星形细胞肿胀 ${ }^{[1]}$, 而使君子酸可以同时激动 AMPA 受体和 trans-ACPD 受体 ${ }^{[4]}$, 因此本实验分别用 AMPA 和 trans-ACPD 与星形细胞作 用, 从受体角度研究谷氨酸引起星形细胞肿胀的机制, 并观察细胞内、外 $\mathrm{Ca}^{2+}$ 在谷氨酸引起的 细胞肿胀中的作用.

1995-08-18 收稿, 1996-01-22 收修改稿

*卫生部“八五”攻关课题 


\section{1 材料和方法}

\section{1 星形细胞培养}

出生 $3 \mathrm{~d}$ 以内的 Wistar 大鼠断头取脑, 去脑膜, 将机械分散的脑细胞悬液加含 $20 \%$ 小牛 血清的 MEM 培养基, 置 $37^{\circ} \mathrm{C}$ 二氧化碳培养箱培养. 约 $10 \mathrm{~d}$ 后将细胞传至 24 孔培养板, 再融 合后镜下观察主要为扁平的星形细胞,胶质纤维酸性蛋白(GFAP)免疫细胞化学染色, 阳性细 胞占 $90 \%$ 以上, 此时换为含有 $0.25 \mathrm{mmol} / \mathrm{L}$ 的二酰丁环腺苷酸 (dBcAMP) 和 $10 \%$ 小牛血清的 MEM 培养基, 每 3 4 d换 1 次液, $10 \mathrm{~d}$ 后相差显微镜下观察, 细胞产生大量突起, 类似纤维型 星形细胞, 用于实验.

\section{2 细胞稱育液中加 EAA 受体激动剂、拮抗剂或药物}

培养细胞用无糖 Earle 氏液洗 3 遍后, 加该液体 $0.5 \mathrm{~mL}$ 于二氧化碳培养箱中 $37^{\circ} \mathrm{C}$ 孵育, 2 $\mathrm{h}$ 后取出, 弃去 Earle 氏液, 加入含谷氨酸受体激动剂的 Earle 氏液 $0.45 \mathrm{~mL}$, 放人二氧化碳培 养箱中 $37^{\circ} \mathrm{C}$ 孵育. L-2-氨基-3-磷酰基丁酸 ( L-AP3) 在加激动剂之前 $1 \mathrm{~h}$ 加入 ${ }^{[5]} . \mathrm{CdCl}_{2}$ 及胆 罗啉与谷氨酸同时加入餒育液中. 无糖 Earle 氏液的配方为 $(\mathrm{mmol} / \mathrm{L}): \mathrm{NaCl} 116, \mathrm{KCl} 5.4$, $\mathrm{CaCl}_{2} 1.8, \mathrm{MgSO}_{4} 0.8, \mathrm{NaH}_{2} \mathrm{PO}_{4} 0.9, \mathrm{NaHCO}_{3} 16.7, \mathrm{HEPES} 20, \mathrm{pH}=7.4$. 无钻 Earle 氏液 仅撤去溶液中的 $\mathrm{CaCl}_{2}$. 进行 $\mathrm{CdCl}_{2}$ 实验时, 平衡盐溶液换为 $(\mathrm{mmol} / \mathrm{L}): \mathrm{NaCl} 140, \mathrm{KCl} \mathrm{5}$, $\mathrm{CaCl}_{2} 1, \mathrm{MgCl}_{2} 1$, HEPES 20, $\mathrm{pH}=7.4$.

\section{3 细胞水含量的测定}

根据 Kletzien 等人的 $\left[{ }^{3} \mathrm{H}\right]$-3-氧-甲基-D-葡萄糖 (OMG) 方法 ${ }^{[6]}$ 测定细胞水含量．培养细 胞与激动剂孵育 $30 \mathrm{~min}$ 后, 在孵育液中加入 $1 \mathrm{mmol} / \mathrm{L}\left[{ }^{3} \mathrm{H}\right] \mathrm{OMG}(50 \mu \mathrm{Ci} / \mathrm{mL}) 0.05 \mathrm{~mL}$, 继 续放入二氧化碳培养箱中孵育, $30 \mathrm{~min}$ 后取出弃去孵育液, 用含 $1 \mathrm{mmol} / \mathrm{L}$ 根皮素(Phloretin) 的冷 Earle 氏液快速洗 3 遍终止反应. 加 $0.2 \mathrm{~mol} / \mathrm{L} \mathrm{NaOH}$ 消化, $10 \mathrm{~min}$ 后取 $0.5 \mathrm{~mL}$ 用于液 体闪炼计数, 取 $0.1 \mathrm{~mL}$ 用于蛋白质含量测定 (Lowry 氏法), 根据 Kletzien 的方法换算成细胞 内水含量, 结果以 $\mu \mathrm{L} / \mathrm{mg}$ 蛋白表示. 实验数据用均数 \pm 标准差表示, 用两样本均数的 $t$ 检验 做统计学分析.

\section{4 动物及药物来源}

动物由中国医学科学院实验动物研究所提供. AMPA, trans-ACPD, L-AP3, 胆罗啉, 根皮 素均购于美国 Sigma 公司, $\left[{ }^{3} \mathrm{H}\right] \mathrm{OMG}$ 购于英国 Amersham 公司, L-谷氨酸钠购于中国科学院 上海生物化学研究所.

\section{2 结果}

\section{1 谷氨酸受体激动剂对培养的星形细胞水含量的影响}

$0.5 \mathrm{mmol} / \mathrm{L}$ 谷氨酸 $1 \mathrm{~h}$ 即可以引起培养的星形细胞水含量增加 $(p<0.05)$, 随着谷氨酸 的剂量增大, 细胞水含量增加幅度加大 (表 1), 相差显微镜可见细胞核肿胀而清晰. 胞体和细 胞突起轮廓消失. iGluR 激动剂 AMPA $1 \mathrm{mmol} / \mathrm{L}$ 不引起细胞水含量的增加, 而 $\mathrm{mGluR}$ 激动 剂 trans-ACPD $1 \mathrm{mmol} / \mathrm{L}$ 可以引起细胞水含量的增加 ( $p<0.05$, 表 1$)$.

\section{2 mGluR 拮抗剂对星形细胞肿胀的影响}

L-AP3 是 mGluR 拮抗剂, $1 \mathrm{mmol} / \mathrm{L}$ L-AP3不影响细胞的水含量, 但是可以拮抗 $1 \mathrm{mmol} / \mathrm{L}$ 


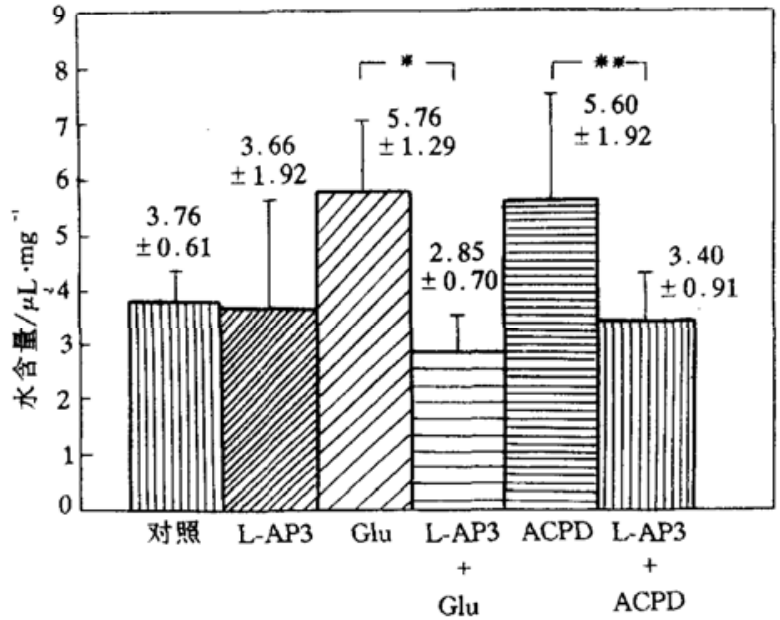

图 1 L-AP3 对谷氨酸受体激动剂引起的细胞 肿胀的影响

与相应的激动剂处理组比较 $* p<0.01, * * p=0.011$

2.3 细胞外 $\mathrm{Ca}^{2+}$ 在谷虽酸引起星形细胞肿胀 中的作用

撤除 Earle 氏溶液中的 $\mathrm{Ca}^{2+}, 1 \mathrm{mmol} / \mathrm{L}$ 的 谷氨酸不再引起细胞的水含量增加 (见表 2), $20 \mu \mathrm{mol} / \mathrm{L}$ 的 $\mathrm{CdCl}_{2}$ 不能阻断 $1 \mathrm{mmol} / \mathrm{L}$ 谷氨 酸引的细胞水含量增加, 但是 $300 \mu \mathrm{mol} / \mathrm{L}$ 的 $\mathrm{CdCl}_{2}$ 可以减少谷䀜酸引起的细胞水含量增加 $(p<0.01$, 图 2).

表 2 细胞外 $\mathrm{Ca}^{2+}$ 对谷穻酸引起的 细胞肿胀的影响 ${ }^{\mathrm{a}}$

\begin{tabular}{|c|c|c|c|}
\hline & \multicolumn{2}{|r|}{ 对照组 } & 谷雬酸组 \\
\hline & $n$ & 水含曾 $/ \mu \mathrm{L} \cdot \mathrm{mg}^{-1}$ & $n$ 水含量 $/ \mu \mathrm{L} \cdot \mathrm{mg}^{-1}$ \\
\hline $2+/ \mathrm{mmol} \cdot \mathrm{L}^{-1} 1.8$ & 8 & $3.76 \pm 0.61$ & $95.76 \pm 1.29^{*} \#$ \\
\hline 0 & 10 & $2.91 \pm 0.90$ & $\begin{array}{ll}9 & 2.48 \pm 0.68\end{array}$ \\
\hline
\end{tabular}

a) 与对照组比较, * $p<0.01$; 与无 $\mathrm{Ca}^{2}+$ 组比较, \# $p<0.01$
trans-ACPD 引起的细胞水含量增加 $(p=$ $0.011)$, 同时也可以拮抗 $1 \mathrm{mmol} / \mathrm{L}$ 谷氨酸引 起的细胞水含量增加 $(p<0.01$, 图 1).

表 1 谷氨酸受体激动剂对培养的 星形细胞水含量的影响 ${ }^{\mathrm{a})}$

\begin{tabular}{|c|c|c|c|}
\hline 分 组 & 剂量/ $/ \mathrm{mmol} \cdot \mathrm{L}^{-1}$ & $n$ & 水含量 $/ \mu \mathrm{L} \cdot \mathrm{mg}^{-1}$ \\
\hline 对 照 & - & 8 & $3.76 \pm 0.61$ \\
\hline \multirow[t]{3}{*}{ 谷䇖酸 } & 0.5 & 7 & $4.92 \pm 1.05^{*}$ \\
\hline & 1 & 9 & $5.76 \pm 1.29^{\circ}$ \\
\hline & 10 & 7 & $6.09 \pm 2.13^{\circ}$ \\
\hline 对 照 & - & 10 & $2.91 \pm 0.90$ \\
\hline AMPA & 1 & 7 & $2.62 \pm 0.45$ \\
\hline \multirow[t]{2}{*}{ trans-ACPD } & 0.1 & 8 & $3.13 \pm 1.01$ \\
\hline & 1 & 8 & $5.60 \pm 1.92^{*}$ \\
\hline
\end{tabular}

a)与对照组比较, * $p<0.05$

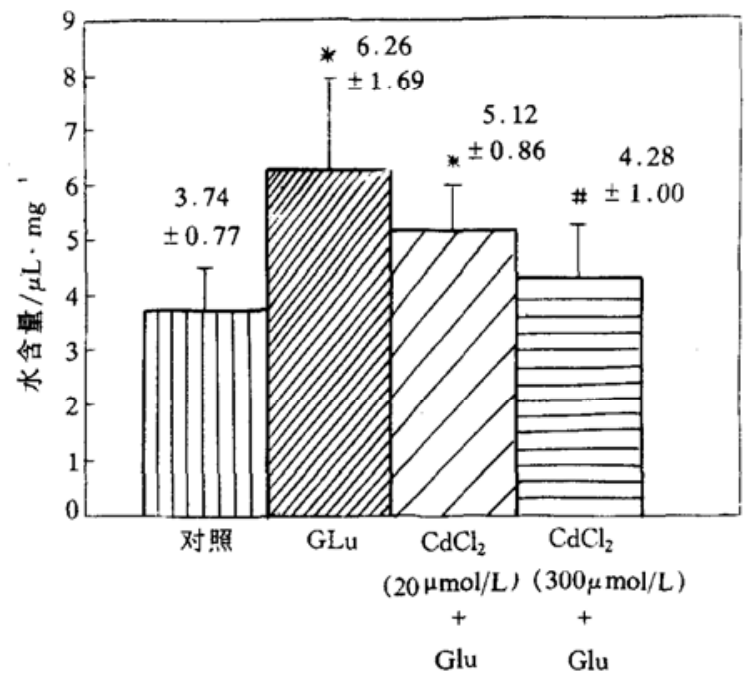

图 2 钙通道阻断剂对谷姿酸引起的 细胞肿胀的影响

与对照组比较* $p<0.01 ;$ 与谷氨酸 处理组比较, \# $p<0.01$

\section{4 细胞内 $\mathrm{Ca}^{2+}$ 在谷氨酸引起星形细胞肿胀中的作用}

胆罗啉用 $0.5 \%$ 二甲基亚砜 (DMSO) 助溶. 实验表明 $0.5 \% \mathrm{DMSO}$ 及 $30 \mu \mathrm{mol} / \mathrm{L}$ 胆罗星 形细胞的水含量没有影响,但是 $30 \mu \mathrm{mol} / \mathrm{L}$ 胆罗啉可以减少谷氨酸引起的星形细胞水含量增 加 $(p<0.05$, 图 3).

\section{3 讨论}

星形细胞是中枢神经系统中数量最多的一种, 在维持大脑正常生理活动中起重要作用. 
在脑缺血、脑外伤等情况下, 星形细胞肿胀是 最早出现、最重要的病理现象之一 ${ }^{[7]}$, 星形细 胞肿胀加重脑水肿, 引起㐿内压增高危及生 命. 文献报道谷氨酸可以引起星形细胞肿 胀 $^{[1]}$, 我们的实验结果证实了这点, 并证明随 着剂量增大, 作用增强.

分析谷氨酸引起星形细胞肿胀的机制可 能有以下几种: 第一, 谷氨酸通过 NMDA 受体 开放钻通道, $\mathrm{Ca}^{2+}$ 大量内流引起细胞肿胀, 但 是文献报道 NMDA 不能引起星形细胞肿胀, NMDA 受体的特异性拮抗剂也不能阻断谷氨 酸引起的细胞肿胀 ${ }^{[1]}$, 而且现在还没有证据表 明星形细胞上有 NMDA 受体, 因此这一假设 不成立. 第二, 谷氨酸通过 KA/AMPA 受体 引起 $\mathrm{Na}^{+}$内流及细胞膜去极化, 进一步使电压

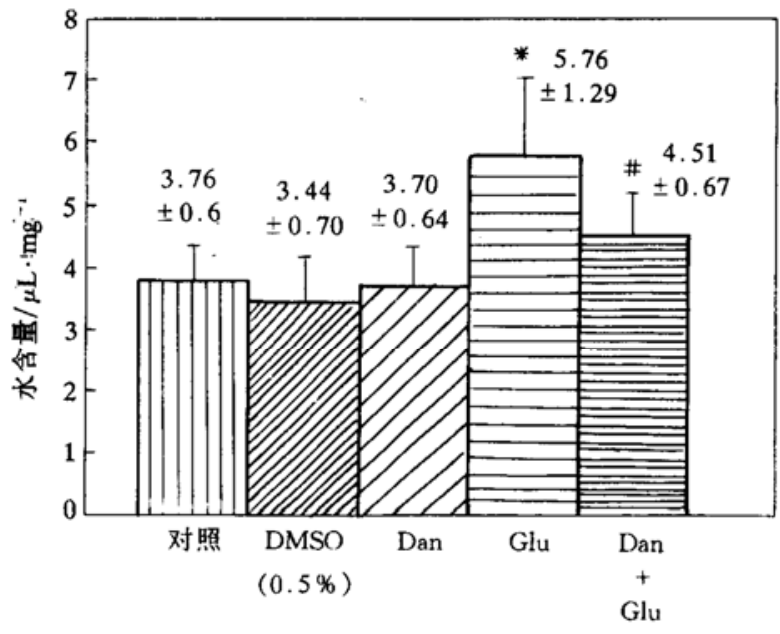

图 3 胆罗啉(Dan)对谷氨酸引起的细胞 肿胀的影响

与对照组比较 * $p<0.01$, 与谷氮酸处理 组比较, \# $p<0.05$

依赖性离子通道开放, $\mathrm{Na}^{+}, \mathrm{Cl}^{-}$内流, 引起细胞肿胀. 已知谷氨酸, $\mathrm{KA}, \mathrm{AMPA}$ 均可以引起培 养的星形细胞膜去极化 ${ }^{[2]}$, 但是我们的实验结果表明 AMPA 不引起星形细胞肿胀, 文献报道 $\mathrm{KA}$ 也不引起星形细胞肿胀 ${ }^{[1]}$, AMPA 受体的拮抗剂不能阻断谷氨酸引起的星形细胞肿胀 ${ }^{[8]}$, 说明谷氨酸引起细胞膜去极化并不能直接引起细胞肿胀. 第三, 谷氨酸通过 mGLuR 激动引 起细胞肿胀. mGluR 激动剂 trans-ACPD 刺激培养的星形细胞水解肌醇磷脂, 产生三磷酸肌 醇 $\left(\mathrm{IP}_{3}\right)$ 和二酰甘油 ( $\left.\mathrm{DG}\right), \mathrm{IP}_{3}$ 又可以引起细胞内钙库释放钻离子 ${ }^{[9]}$. 我们的实验结果表明 trans-ACPD 可以引起培养的星形细胞肿胀, L-AP3 可以拮抗 trans-ACPD 引起的细胞肿胀, 同 时也可以拮抗谷氨酸引起的细胞肿胀, 提示谷氨酸能通过 mGluR 引起细胞肿胀. 用胆罗啉抑 制细胞内钙库释放 $\mathrm{Ca}^{2+}[10]$, 可以减轻谷氨酸引起的星形细胞肿胀, 证实细胞内 $\mathrm{Ca}^{2+}$ 在谷氨酸 引起的细胞肿胀中起一定的作用. 因此谷氨酸引起的星形细胞肿胀可能是由 mGluR 激动导 致胞内一系列信使物质 $\mathrm{IP}_{3}$ 和 $\mathrm{Ca}^{2+}$ 等的变化所介导的. 本文首次报道胆罗啉可以减轻谷氨 酸引起的星形细胞肿胀, 并明确提出 mGLuR 激动引起星形细胞肿胀.

钻在细胞体积调节中起重要作用. 实验发现撤除平衡盐溶液中的 $\mathrm{Ca}^{2+}$ 后, 谷氨酸不再引 起星形细胞肿胀, 说明谷氨酸引起的细胞肿胀是钙依赖性的, 这与文献报道相符 ${ }^{[11]}$. 低浓度 的 $\mathrm{CdCl}_{2}$ 可阻断 $\mathrm{L}$ 型和 $\mathrm{N}$ 型钙通道, 高浓度的 $\mathrm{CdCl}_{2}$ 可阻断 $\mathrm{L}$ 型、 $\mathrm{N}$ 型和 $\mathrm{T}$ 型钻通道 ${ }^{[12]}$, 我 们用 $20 \mu \mathrm{mol} / \mathrm{L}$ 的 $\mathrm{CaCl}_{2}$ 未能减轻谷氨酸引起的星形细胞肿胀, 说明仅阻断 $\mathrm{L}$ 型、 $\mathrm{N}$ 型钻通道 并不能减轻谷氨酸的作用; 而用 $300 \mu \mathrm{mol} / \mathrm{L}$ 的 $\mathrm{CdCl}_{2}$ 可以减轻谷氨酸引起的细胞肿胀, 提示 肿胀时细胞外 $\mathrm{Ca}^{2+}$ 内流可能是通过 $\mathrm{T}$ 型钙通道完成的. 这与 Hansson 的报道略有不同, 她认 为 $\mathrm{L}$ 型、 $\mathrm{N}$ 型和 $\mathrm{T}$ 型钙通道不参与谷氨酸引起的星形细胞肿胀 ${ }^{[13]}$.

综上所述, mGluR 激动可能在谷氨酸引起星形细胞肿胀中起重要作用. 细胞外 $\mathrm{Ca}^{2+}$ 内流 以及细胞内钙库释放 $\mathrm{Ca}^{2+}$ 在细胞肿胀的发生、发展中都起一定的作用. 因此治疗细胞性脑水 肿的策略可以包括使用 mGluR 拮抗剂和作用于 $\mathrm{T}$ 型钻通道的拮抗剂. 
致谢 吉宏龙进行了 GFAP 免疫细胞化学染色, 刘淑英、徐立新、杨晶晶、郑淑荣在实验 中给予大力支持, 在此一并感谢.

\section{参 考 文 献}

1 Chan P H, Chu L, Chen S. Effects of MK-801 on glutamate-induced swelling of astrocytes in primary cell culture. J Neurosci Res, 1990, 25: 87 93

2 Backus K H, Kettenmann H, Schachner M. Pharmacological characterization of the glutamate receptor in cultured astrocytes. J Neurosci Res, 1989, 22: 274 282

3 Monaqhan D T, Bridges R J, Cotman C W. The excitatory amino acid receptors: their classes, pharmacology, and distinct properties in the function of central nervous system. Annu Rev Pharmacol Toxicol, 1989, 29: 365 402

4 Murphy S N, Miller R J. Two distict quisqualate receptors regulate $\mathrm{Ca}^{2+}$ homeostasis in hippocampal neurons in vitro. Mol Pharmacol, 1989, 35: 671 680

5 Littman L, Robinson M B. The effects of L-glutamate and trans-( \pm )-1-amino-1,3-cyclopentanedicarboxylate on phosphoinsitide hydrolysis can be pharmacologically differentiated. J Neurochem, 1994, 63: $1291 \sim 1302$

6 Kletzien R F, Pariza M W, Becker J E et al. A method using 3-O-methyl-D-glucose and phloretin for the determination of intracellular water space of cells in monolayer culture. Anal Biochem, 1975, 68: 537 544

7 Kimelberg H K, Ransom B R. Physiological and pathological aspects of astrocytic swelling. In: Fedoroff S, Vemadakis A eds. Astrocytes, Cell Biology and Pathology of Astrocytes, Ve. New York: Academic Press, 1986. 129 166

8 Hansson E, Johansson B B, Westergren I et al. Glutamate-induced swelling of single astroglial cells in primary culture. Neuroscience, 1994, 63:1057 1066

9 Cornell-Bell A H, Finkbeiner S M. Ca ${ }^{2+}$ waves in astrocytes. Cell Calcium, 1991, 12: 185 204

10 Frandsen A, Schousboe A. Mobilization of dantrolene-sensitive intracellular calium pools is involved in the cyttoxicity induced by quisqualate and N-methyl-D-aspartate but not by 2-amino-3-(3-hydroxy-5-methylisoxazaol-4-yl) propionate and kainate in cultured cerebral cortical neurons. Proc Natl Acad Sci USA; 1992, 89: 2 590 2 594

11 Koyama Y, Baba A, Iwata H. L-glutamate-induced swelling of cultured astrocytes is dependent on extracellular $\mathrm{Ca}^{2+}$. Neurosci Lett, 1991, 122: 210 212

12 Tsien R W, Fox A P, Hess P et al. Multiple types of calcium channal in excitable cells. In: Hille E, Fambrough D M eds. Proteins of Excitable Membranes. New York: Wiley-Interscience, 1987. 167 187

13 Hansson. Metabotropic glutamate receptor activation induces astroglial swelling. J Bio Chem, 1994, 269: $21955 \sim 21961$ 